\title{
Atom Probe Tomography Studies of GaN-Based Semiconductor Materials
}

\author{
S.E. Bennett, ${ }^{*}$ R.A. Oliver, ${ }^{*}$ D.W. Saxey, ${ }^{* *}$ A. Cerezo, ${ }^{* *}$ P.H. Clifton, ${ }^{* * *}$ R.M. Ulfig, ${ }^{* * *}$ \\ M.J. Kappers, ${ }^{*}$ and C.J. Humphreys* \\ * Department of Materials Science and Metallurgy, University of Cambridge, Pembroke Street, \\ Cambridge, CB2 3QZ, UK \\ ** Department of Materials, University of Oxford, Parks Road, Oxford, OX1 3PH, UK \\ *** Imago Scientific Instruments Corporation, 5500 Nobel Drive, Madison, WI 53711
}

$\mathrm{GaN}$ is a semiconductor used in light emitting diodes (LEDs) and laser diodes. Here, atom probe tomography is used to investigate two GaN-based materials: first, a p-type AlGaN/GaN superlattice and second, an InGaN/GaN quantum well sample that has shown particularly high internal quantum efficiency (IQE) in the green spectral region [1].

P-type conducting layers in GaN-based devices are often realized using Mg doping, which suffers from intrinsically low doping efficiency. The charge carrier concentration can be enhanced using alternating layers of $\mathrm{Mg}$-doped $\mathrm{AlGaN}$ and $\mathrm{GaN}$, known as a p-type superlattice, to engineer the band structure and obtain significantly increased hole concentration. An AlGaN/GaN superlattice was studied using SIMS, which suggested a difference in doping density between the two materials. Atom probe tomography was then used to investigate the $\mathrm{Mg}$ distribution. Areas of increased $\mathrm{Mg}$ concentration were observed in both the $\mathrm{GaN}$ and $\mathrm{AlGaN}$ layers, although there were significantly more of these 'clusters' present in the AlGaN. Figure 1 shows these clusters within the layers using isoconcentration surfaces at 4\% $\mathrm{Mg}$ (small pink clusters) and 7\% Al (blue layers) and a clipping in the y-dimension. High resolution TEM lattice imaging was used to investigate the structure of the $\mathrm{Mg}$ clusters and pyramidal defects like those reported in the literature were clearly visible in the AlGaN layers [2]. Structural and compositional comparisons of the $\mathrm{Mg}$ clustering were made between the TEM and atom probe data.

Green-emitting InGaN-based devices show significantly lower IQE values when compared to blue emitters. Closing this 'green gap' is a common goal of researchers in the field. A particularly high efficiency for green (12\% IQE), has been reported in a $10 \mathrm{InGaN}$ quantum well sample that showed gross well-width fluctuations in TEM observations [1]. This material was studied by atom probe to further investigate the 3D morphology and composition of the quantum wells, in the hope that a cause for its increased efficiency could be determined and exploited in the future. Using a $4 \%$ In isoconcentration surface, it can be seen in Figure 2A that the quantum wells show highly nonuniform thicknesses. Figure $2 \mathrm{~B}$ shows the bottom well with an extended low indium region running through the well. It is thought that these gaps may help prevent carrier diffusion to dislocations, leading to the high observed IQE.

\section{References}

[1] P.M.F.J. Costa et al., Phys. Stat. Sol. (a). 203 (7) (2006) 1729.

[2] P. Vennegues et al., App. Phys. Lett. 77 (6) (2000) 880.

[3] This research was supported by the Engineering and Physical Sciences Research Council, Sharp Laboratories of Europe and Pembroke College, Cambridge. 


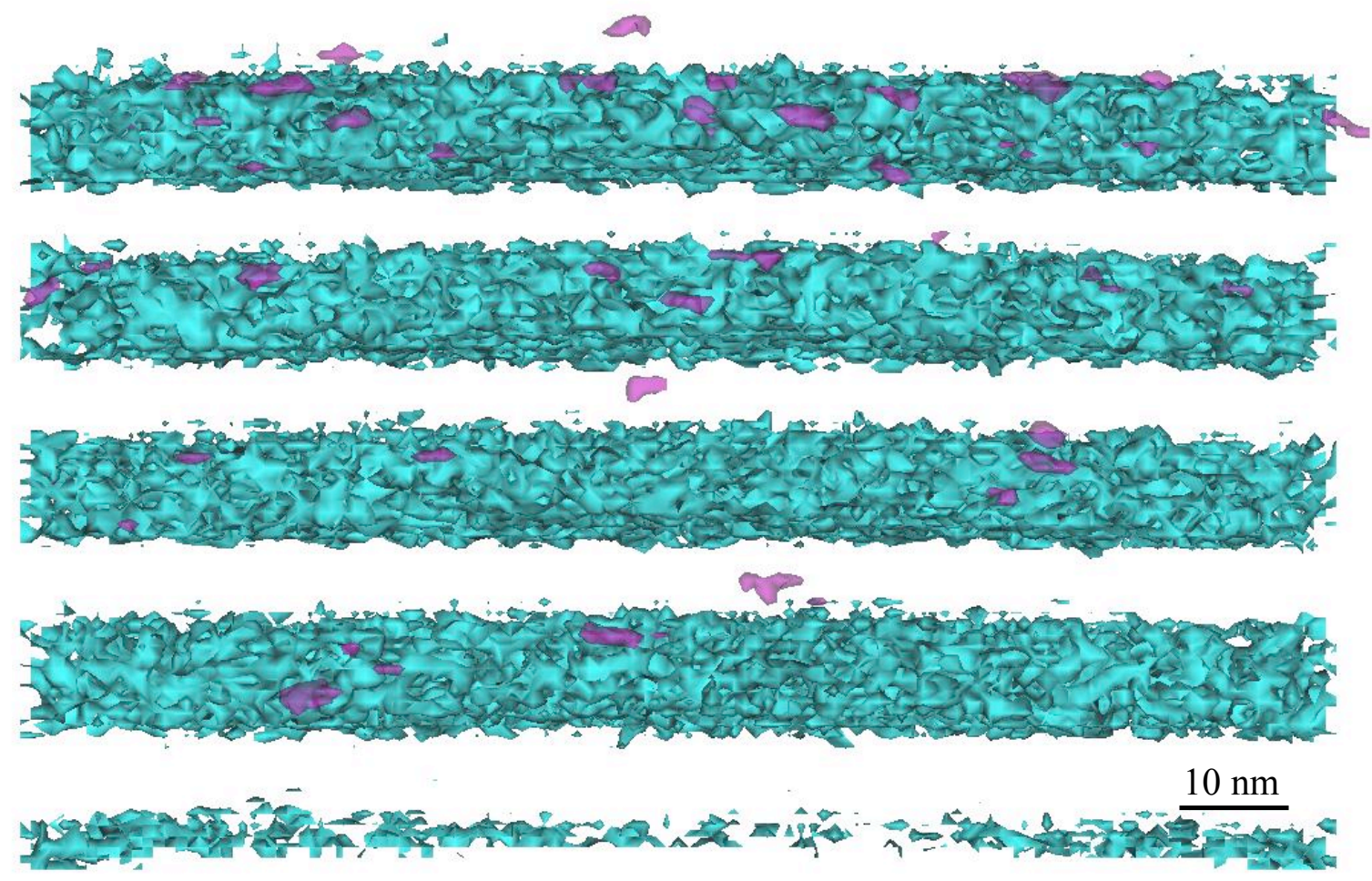

FIG. 1. Mg-doped $\mathrm{AlGaN} / \mathrm{GaN}$ superlattice, with isoconcentration surfaces at $4 \% \mathrm{Mg}$ (small pink clusters) and $7 \% \mathrm{Al}$ (blue layers). A y-axis clipping is shown to expose some Mg clusters, which are found primarily in the AlGaN.
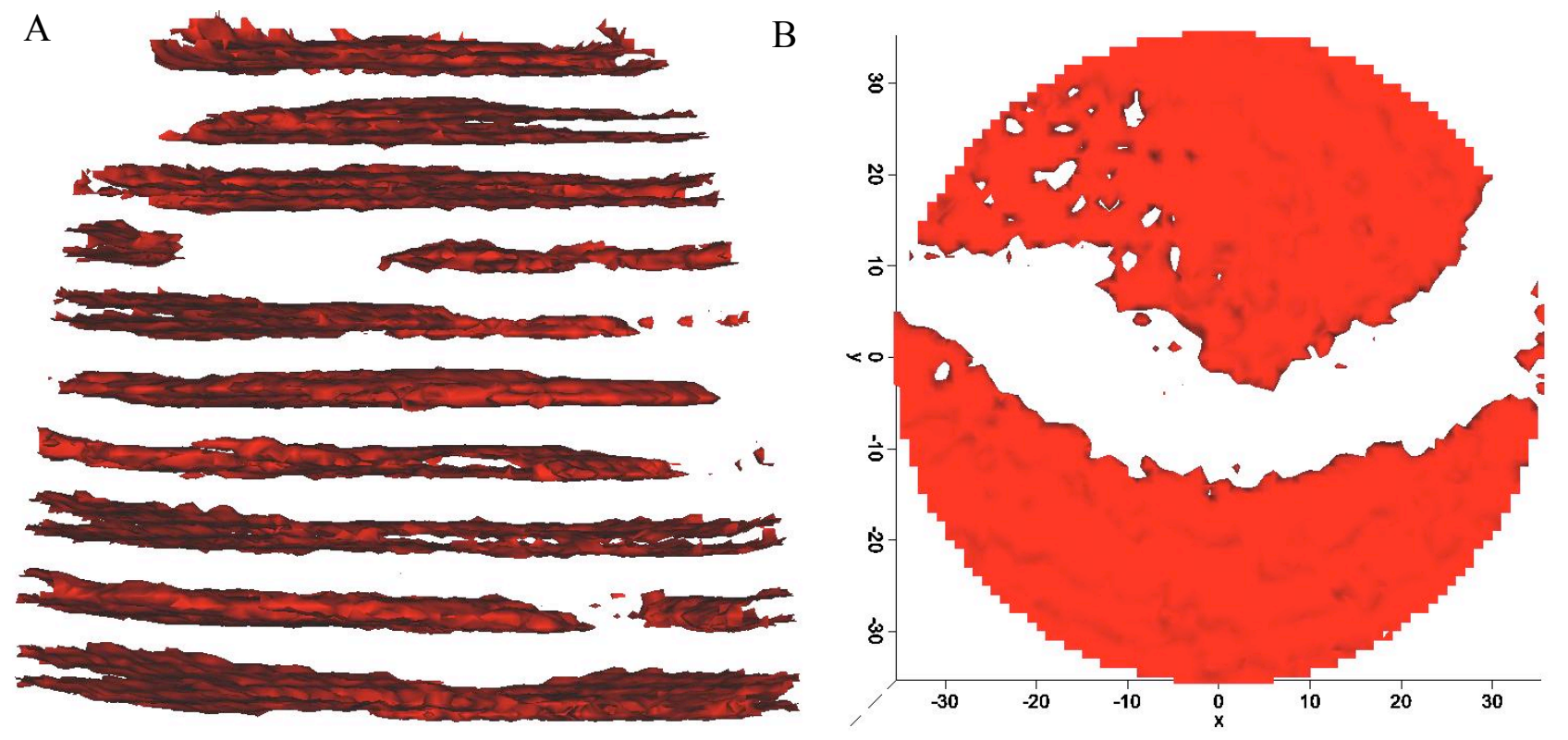

FIG. 2. Green-emitting InGaN multiple quantum well, with gross well-width fluctuations. An isoconcentration surface is shown at 4\% In. A. Full quantum well stack. B. Top-view of the bottom layer, showing an extended low indium content region running through the well. Scale bars are in nm. 